\title{
Optimal design of Purcell's three-link swimmer
}

\author{
Laetitia Giraldi, ${ }^{1, *}$ Pierre Martinon, ${ }^{2}$ and Marta Zoppello ${ }^{3}$ \\ ${ }^{1}$ UMA, ENSTA-Paristech, Palaiseau, France \\ ${ }^{2}$ INRIA Saclay and CMAP Ecole Polytechnique, Palaiseau, France \\ ${ }^{3}$ Department of Mathematics, University of Padova, Padova, Italy
}

(Received 8 September 2014; published 13 February 2015)

\begin{abstract}
In this paper we address the question of the optimal design for the Purcell three-link swimmer. More precisely, we investigate the best link length ratio which maximizes its displacement. The dynamics of the swimmer is expressed as an ordinary differential equation, using the resistive force theory. Among a set of optimal strategies of deformation (strokes), we provide an asymptotic estimate of the displacement for small deformations, from which we derive the optimal link ratio. Numerical simulations are in good agreement with this theoretical estimate and also cover larger amplitudes of deformation. Compared with the classical design of the Purcell swimmer, we observe a gain in displacement of roughly $60 \%$.
\end{abstract}

DOI: 10.1103/PhysRevE.91.023012

The study of self-propulsion at microscopic scale is attracting increasing attention in the recent literature both because of its intrinsic biological interest and for the possible implications on the design of bioinspired artificial replicas reproducing the functionalities of biological systems (see, for instance, [1-4]). At this scale, inertia forces are negligible compared to the viscous ones, i.e., low Reynolds number, calling for different swimming strategies than at greater scales. Thus, we assume that the surrounding fluid is governed by Stokes equations, which implies that hydrodynamic forces and torques are linear with respect to the swimmer's velocity. In the case of planar flagellar propulsion, the resistive force theory (RFT) provides a simple and concise way to compute a local approximation of hydrodynamic forces and Newton laws (see [5]). The resulting equations can be written as a system of linear ordinary differential equations (ODEs) (see [6-8]). In this paper we focus on one of the first example of microswimmer model found in literature: the "three-link swimmer" [9]. This model is still attracting interest in recent studies; see [10,11]. The structure of the equations of motion leads to establishing a connection between geometrical control theory and microswimming (see [12]). In this paper, we address the optimal design issue, namely finding the optimal length ratio between the three links which maximizes displacement of the swimmer. A similar issue has been studied in [13], where a Fourier expansion is used to derive an optimal design. Here techniques from the control theory are used to approximate the leading order term of the swimmer's displacement. Maximizing this leading term gives a theoretical value for the optimal link ratio. This procedure could be applied to others models such as the three-sphere swimmer (see [14]).

The paper is organized as follows. Section I recalls the equations of motion for the Purcell swimmer. Section II presents strokes which maximize the $x$ displacement, based on previous simulations from [8]. Section III details the expansion of the displacement for such strokes at small amplitude. By maximizing the leader term of this expansion, we derive an optimal length ratio. Section IV shows the numerical simulations whose results are consistent with this theoretical ratio, for both small and large amplitude of deformation.

${ }^{*}$ Corresponding author: laetitia.giraldi@polytechnique.edu
PACS number(s): 47.63.Gd, 02.30.Yy

\section{MODELING}

Purcell's three-link swimmer. The three-link swimmer is modeled by the position of the center of the second stick $\mathbf{x}=(x, y)$, the angle $\theta$ between the $x$ axis and the second stick (the orientation of the swimmer). The shape of the swimmer is defined by the two relative angles $\beta_{1}$ and $\beta_{3}$ (see Fig. 1). We also denote by $L$ and $L_{2}$ the length of the two external arms and central link.

Dynamics via resistive force theory. We approximate the nonlocal hydrodynamic forces exerted by the fluid on the swimmer with local drag forces depending linearly on the velocity. We denote by $\mathbf{e}_{i}^{\|}$and $\mathbf{e}_{i}^{\perp}$ the unit vectors parallel and perpendicular to the $i$ th link, and we also introduce $\mathbf{v}_{i}(s)$, the velocity of the point at distance $s$ from the extremity of the $i$ th link; that is,

$$
\begin{aligned}
& \mathbf{v}_{1}(s)=\dot{\mathbf{x}}-\frac{L_{2}}{2} \dot{\theta} \mathbf{e}_{2}^{\perp}-s\left(\dot{\theta}-\dot{\beta}_{1}\right) \mathbf{e}_{1}^{\perp}, \quad s \in[0, L], \\
& \mathbf{v}_{2}(s)=\dot{\mathbf{x}}-\left(s-\frac{L_{2}}{2}\right) \dot{\theta} \mathbf{e}_{2}^{\perp}, \quad s \in\left[0, L_{2}\right], \\
& \mathbf{v}_{3}(s)=\dot{\mathbf{x}}+\frac{L_{2}}{2} \dot{\theta} \mathbf{e}_{2}^{\perp}+s\left(\dot{\theta}-\dot{\beta}_{3}\right) \mathbf{e}_{3}^{\perp}, \quad s \in[0, L] .
\end{aligned}
$$

The force $\mathbf{f}_{i}$ acting on the $i$ th segment is taken as

$$
\mathbf{f}_{i}(s):=-\xi\left[\mathbf{v}_{i}(s) \cdot \mathbf{e}_{i}^{\|}\right] \mathbf{e}_{i}^{\|}-\eta\left[\mathbf{v}_{i}(s) \cdot \mathbf{e}_{i}^{\perp}\right] \mathbf{e}_{i}^{\perp},
$$

where $\xi$ and $\eta$ are, respectively, the drag coefficients in the directions of $\mathbf{e}_{i}^{\|}$and $\mathbf{e}_{i}^{\perp}$.

Neglecting inertia forces, Newton laws are written as

$$
\mathbf{F}=0, \quad \mathbf{e}_{z} \cdot \mathbf{T}_{\mathbf{x}}=0,
$$

where $\mathbf{F}$ is the total force exerted on the swimmer by the fluid,

$$
\mathbf{F}=\sum_{i=1}^{N} \int_{0}^{L_{i}} \mathbf{f}_{i}(s) d s,
$$

and $\mathbf{T}_{\mathbf{x}}$ is the corresponding total torque computed with respect to the central point $\mathbf{x}$,

$$
\mathbf{T}_{\mathbf{x}_{1}}=\sum_{i=1}^{N} \int_{0}^{L_{i}}\left[\mathbf{x}_{i}(s)-\mathbf{x}_{1}\right] \times \mathbf{f}_{i}(s) d s .
$$




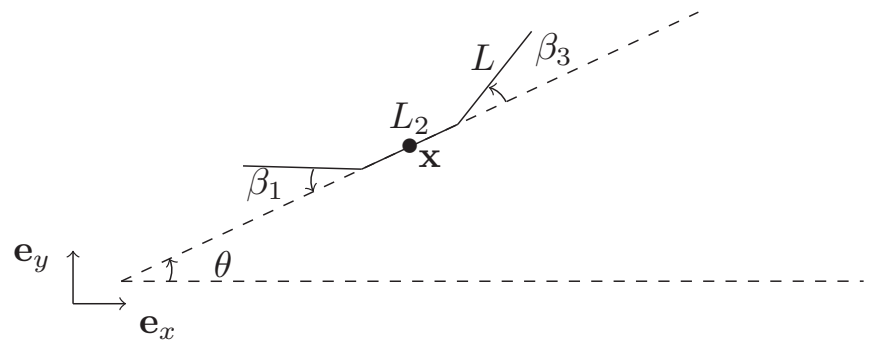

FIG. 1. Purcell's three-link swimmer.

Since the $\mathbf{f}_{i}(s)$ are linear in $\dot{\mathbf{x}}, \dot{\theta}, \dot{\beta}_{1}, \dot{\beta}_{3}$, the system (2) can be rewritten as

$$
\mathbf{A}(\mathbf{z}) \cdot\left(\begin{array}{c}
\dot{\mathbf{x}} \\
\dot{\theta}
\end{array}\right)-\mathbf{B}(\mathbf{z}) \cdot\left(\begin{array}{c}
\dot{\beta}_{1} \\
\dot{\beta}_{3}
\end{array}\right)=0,
$$

where $\mathbf{z}(t):=\left(\beta_{1}, \beta_{3}, x, y, \theta\right)(t)^{T}$. The matrix $\mathbf{A}$ is known as the "grand resistance matrix" and is invertible (see [6]). Then the dynamics of the swimmer is finally expressed as an ODE system,

$$
\dot{\mathbf{z}}(t)=f\left(\mathbf{z}, \dot{\beta}_{1}, \dot{\beta}_{3}\right)=\mathbf{g}_{1}(\mathbf{z}(t)) \dot{\beta}_{1}(t)+\mathbf{g}_{2}(\mathbf{z}(t)) \dot{\beta}_{3}(t),
$$

where

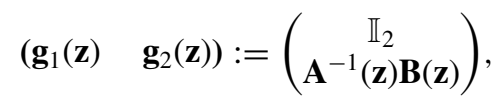

with $\mathbb{I}_{2}$ the $2 \times 2$ identity matrix. The literal expression of the $\mathbf{g}_{i}$ is quite complicated (several pages).

\section{OPTIMAL STROKES}

Optimal control problem. We are interested in finding a periodic sequence of deformations which maximizes the displacement of the swimmer along the $x$ axis. More precisely, we optimize both the link length ratio $L_{2} / L$ and the deformation of the swimmer over time. Taking the deformation speed $\dot{\beta}_{1 \mid 3}$ as control functions, we obtain the optimal control problem

$$
(\mathrm{OCP})\left\{\begin{array}{l}
\max x_{2}(T) \text { s.t. } \\
\dot{\mathbf{z}}(t)=f\left(\mathbf{z}(t), \dot{\beta}_{1}, \dot{\beta}_{3}\right) \quad \forall t \in[0, T] \\
\dot{\beta}_{1 \mid 3} \in \mathbf{U}=[-b, b] \quad \forall t \in[0, T] \\
\beta_{1 \mid 3}(t) \in[-a, a] \quad \forall t \in[0, T] \\
x_{2}(0)=y_{2}(0)=\theta_{2}(0)=0, y_{2}(T)=\theta_{2}(T)=0 \\
\beta_{1 \mid 3}(0)=\beta_{1 \mid 3}(T) \\
2 L+L_{2}=c
\end{array}\right.
$$

We set the constraints $a$ and $b$ over the amplitude and deformation speed, as well as the total length $c$ of the swimmer. The final time $T$ is fixed, and the constraint $\beta_{1 \mid 3}(0)=\beta_{1 \mid 3}(T)$ ensures that the swimmer is in the same configuration at the initial and final times. Note that this condition can be satisfied by either a single stroke or a sequence of strokes. From [8], numerically solving (OCP) typically gives a periodic sequence of identical strokes. Their phase portrait is octagonal, as illustrated on Fig. 2, and we detail how this shape is consistent with optimal control theory.

Pontryagin's maximum principle (PMP). We recall here the PMP as it gives some insight on the shape of optimal strokes. This theorem in optimal control, introduced by Pontryagin

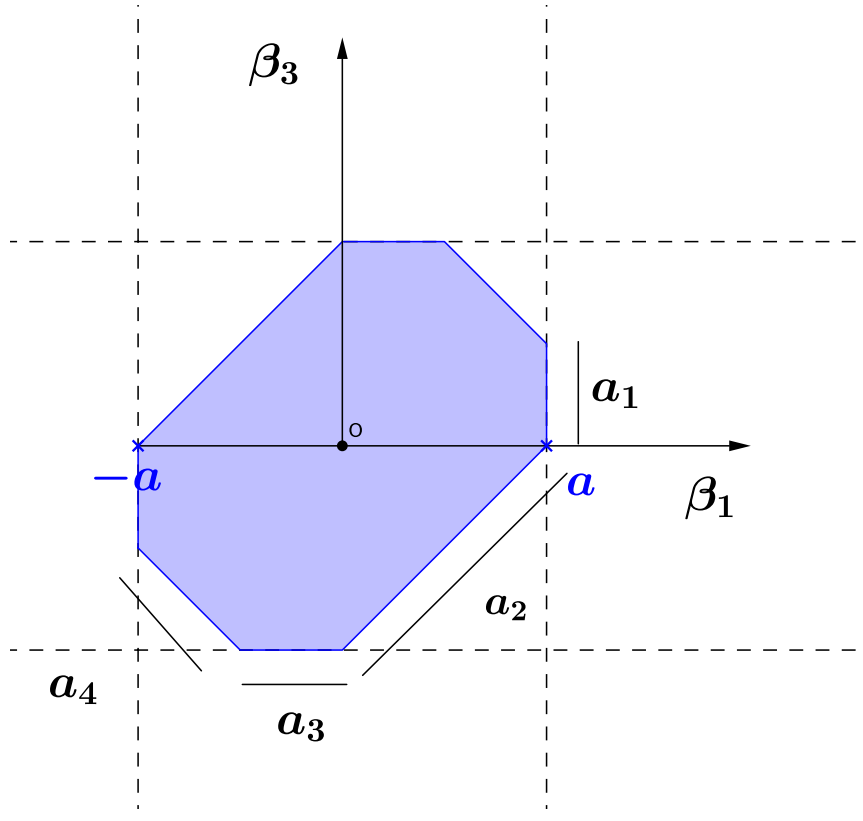

FIG. 2. (Color online) Phase portrait $\left(\beta_{1}, \beta_{3}\right)$ of the octagonal stroke considered for the expansion of the displacement.

et al. in [15], gives necessary conditions for local optimality. Interested readers can find more information on the PMP in $[16,17]$. The PMP is characterized by a Hamiltonian function $H$ that formally depends on the state variables $\mathbf{z}$, the control functions $\dot{\beta}_{1 \mid 3}$, and so-called costate variables noted $\mathbf{p}$. While originally inspired by the Hamiltonian in mechanics, in the context of optimal control $H$ does not actually correspond to the energy of the system. The costate variables play the part of the generalized velocities in Lagrangian mechanics, and they can be interpreted as Lagrange multipliers (in the sense of constrained optimization) related to the dynamics of the system. Let the Hamiltonian be

$$
H\left(\mathbf{z}, \mathbf{p}, \dot{\beta}_{1}, \dot{\beta}_{3}\right)=\left\langle\mathbf{p}, \mathbf{g}_{1}(\mathbf{z})\right\rangle \dot{\beta}_{1}+\left\langle\mathbf{p}, \mathbf{g}_{2}(\mathbf{z})\right\rangle \dot{\beta}_{3} .
$$

Under the assumption that $\mathbf{g}_{1 \mid 2}$ are continuous and $C^{1}$ with respect to $\mathbf{z}$, the PMP states that if $\left(\mathbf{z}^{*}, \dot{\beta}_{1}^{*}, \dot{\beta}_{3}^{*}\right)$ is a solution of (OCP), then there exists $\mathbf{p}^{*} \neq 0$ absolutely continuous such that $\dot{\mathbf{z}}^{*}=H_{p}\left(\mathbf{z}^{*}, \mathbf{p}^{*}, \dot{\beta}_{1}^{*}, \dot{\beta}_{3}^{*}\right), \dot{\mathbf{p}}^{*}=-H_{z}\left(\mathbf{z}^{*}, \mathbf{p}^{*}, \dot{\beta}_{1}^{*}, \dot{\beta}_{3}^{*}\right), \mathbf{p}^{*}(T)$ is orthogonal to the cotangent cone of the final conditions at $\mathbf{z}^{*}(T)$, and $\left(\dot{\beta}_{1}^{*}, \dot{\beta}_{3}^{*}\right)$ maximizes the Hamiltonian for almost every time $t \in[0, T]$.

Bang arcs. The Hamiltonian in (7) is linear in the controls $\dot{\beta}_{1 \mid 3}$. If we assume $\left\langle\mathbf{p}, \mathbf{g}_{i}(\mathbf{z})\right\rangle \neq 0$ for $i=1,2$ over a time interval, then the optimal control $\dot{\beta}_{1 \mid 3^{*}}$ that maximizes $H$ must be on the boundary of $U=\{(-b,-b),(-b, b),(b,-b),(b, b)\}$. In terms of phase portrait, this corresponds to diagonal lines.

Constrained arcs. Moreover, we have the constraints on the joint angles $\beta_{1 \mid 3}(t) \in[-a, a]$. When one of them is active and $\left|\beta_{i}\right|=a$, the corresponding control $\dot{\beta}_{i}=0$. In terms of phase portrait, this gives horizontal or vertical lines.

Symmetries. As stated in [13], we expect optimal strokes to be symmetric with respect to the diagonal axes $\beta_{1}=\beta_{3}$ and $\beta_{1}=-\beta_{3}$. This comes from the equations of motion being linear and time independent. From the linearity, optimal 
strokes should be invariant by reflection with respect to the axis of the swimmer's body. From time independence, the stroke should be invariant when inverting the arms movement and going backwards in time.

\section{OPTIMAL SWIMMER DESIGN}

In this section, we express the leader term of the swimmer's displacement for a stroke of small perimeter which satisfies all properties stated in the previous section. We represent the stroke by a closed octagonal curve $\gamma$ in the phase portrait $\left(\beta_{1}, \beta_{3}\right)$; see Fig. 2 .

As a consequence of neglecting inertia forces, velocities appear linearly in the dynamic, and time can be rescaled without changing the dynamics. Thus, the displacement of the swimmer after one stroke does not depend on the speed along the curve $\gamma$, but only on the shape of the stroke. From now on, we parametrize $\gamma$ by the arc length $s$. Using an approach similar to that in [18], we express the swimmer's displacement along the $x$ axis [i.e., $x(T)-x(0)$ ] as an asymptotic expansion for small length $a_{i}, i=1, \ldots, 4$.

Displacement over the arc $s \in\left[0, a_{1}\right]$. On this part, according to Fig. 2, we set $\mathbf{u}=\left(\dot{\beta}_{1}, \dot{\beta}_{3}\right)=(0,-1)$. The dynamics of the swimmer is therefore given by $\dot{\mathbf{z}}=-\mathbf{g}_{2}$, and the time expansion at order two is given by

$$
\begin{aligned}
\mathbf{z}\left(a_{1}\right)= & \mathbf{z}(0)-a_{1} \mathbf{g}_{2}(\mathbf{z}(0)) \\
& +\left.\frac{a_{1}^{2}}{2} \frac{\partial \mathbf{g}_{2}}{\partial \mathbf{z}}\right|_{\mathbf{z}(0)}\left(\mathbf{g}_{2}(\mathbf{z}(0))\right)+o\left(a_{1}^{3}\right) .
\end{aligned}
$$

Displacement over the arc $s \in\left[a_{1}, a_{1}+a_{2}\right]$. Similarly, the position of the swimmer at $s=a_{1}+a_{2}$ can be expressed as

$$
\begin{aligned}
\mathbf{z}\left(a_{1}+a_{2}\right)= & \mathbf{z}\left(a_{1}\right)-\frac{a_{2} \sqrt{2}}{2} \mathbf{h}\left(\mathbf{z}\left(a_{1}\right)\right) \\
& +\left.\frac{a_{2}^{2}}{4} \frac{\partial \mathbf{h}}{\partial \mathbf{z}}\right|_{\mathbf{z}\left(a_{1}\right)}\left(\mathbf{h}\left(\mathbf{z}\left(a_{1}\right)\right)\right)+o\left(a_{2}^{3}\right),
\end{aligned}
$$

where $\mathbf{h}:=\mathbf{g}_{1}+\mathbf{g}_{2}$. Plugging the value of $\mathbf{z}\left(a_{1}\right)$ from (8) into (9) and neglecting the terms of order greater than two, we get

$$
\begin{aligned}
\mathbf{z}\left(a_{1}+a_{2}\right)= & \mathbf{z}(0)+c_{1}\left(\mathbf{g}_{1}, \mathbf{g}_{2}, \mathbf{z}(0), a_{1}, a_{2}\right) \\
& +c_{2}\left(\mathbf{g}_{1}, \mathbf{g}_{2}, \mathbf{z}(0), a_{1}, a_{2}\right) \\
& +o\left(a_{1}^{3}\right)+o\left(a_{2}^{3}\right),
\end{aligned}
$$

with

$$
\begin{aligned}
c_{1}\left(\mathbf{f}, \mathbf{g}, \mathbf{z}, a_{1}, a_{2}\right)= & -\frac{\sqrt{2} a_{2}}{2} \mathbf{f}(\mathbf{z})+\left(-a_{1}-\frac{\sqrt{2} a_{2}}{2}\right) \mathbf{g}(\mathbf{z}), \\
c_{2}\left(\mathbf{f}, \mathbf{g}, \mathbf{z}, a_{1}, a_{2}\right)= & \left.\frac{a_{2}^{2}}{4} \frac{\partial \mathbf{f}}{\partial \mathbf{z}}\right|_{\mathbf{z}}(\mathbf{f}(\mathbf{z}))+\left.\frac{a_{2}^{2}}{4} \frac{\partial \mathbf{g}}{\partial \mathbf{z}}\right|_{\mathbf{z}}(\mathbf{f}(\mathbf{z})) \\
& +\left.\left(\frac{a_{1} a_{2} \sqrt{2}}{2}+\frac{a_{2}^{2}}{4}\right) \frac{\partial \mathbf{f}}{\partial \mathbf{z}}\right|_{\mathbf{z}}(\mathbf{g}(\mathbf{z})) \\
& +\left.\left(\frac{a_{1} a_{2} \sqrt{2}}{2}+\frac{a_{2}^{2}}{4}+\frac{a_{1}^{2}}{2}\right) \frac{\partial \mathbf{g}}{\partial \mathbf{z}}\right|_{\mathbf{z}}(\mathbf{g}(\mathbf{z})) .
\end{aligned}
$$

Displacement over the complete stroke. Iterating the computations along each arc and noting by $P=2\left(a_{1}+a_{2}+\right.$ $\left.a_{3}+a_{4}\right)$ the stroke perimeter, the expansion of the total displacement for the octagonal stroke is finally obtained as

$$
\mathbf{z}(T)-\mathbf{z}(0)=C\left[\mathbf{g}_{1}, \mathbf{g}_{2}\right](\mathbf{z}(0))+o\left(a_{i}^{3}\right)_{i=1-4},
$$

where

$$
\begin{aligned}
C= & \frac{a_{1} a_{2} \sqrt{2}}{2}+a_{1} a_{3}+\frac{a_{2} a_{3} \sqrt{2}}{2}+\frac{a_{1} a_{4} \sqrt{2}}{2}+a_{2} a_{4} \\
& +\frac{a_{3} a_{4} \sqrt{2}}{2}
\end{aligned}
$$

and

$$
\left[\mathbf{g}_{1}, \mathbf{g}_{2}\right](\mathbf{z}(0))=\nabla \mathbf{g}_{2}(\mathbf{z}(0)) \cdot \mathbf{g}_{1}(\mathbf{z}(0))-\nabla \mathbf{g}_{1}(\mathbf{z}(0)) \cdot \mathbf{g}_{2}(\mathbf{z}(0))
$$

is the Lie brackets of $\mathbf{g}_{1}$ and $\mathbf{g}_{2}$ at point $\mathbf{z}(0)$. Choosing the starting point $\mathbf{z}(0)$ such that $\theta(0)=\beta_{1}(0)=\beta_{3}(0)=0$, we compute the Lie bracket with a formal calculus tool

$$
\left[\mathbf{g}_{1}, \mathbf{g}_{2}\right](0,0, x, y, 0)=\left(\begin{array}{c}
0 \\
0 \\
\frac{\eta-\xi}{\xi} \frac{L^{3} L_{2}\left(3 L+2 L_{2}\right)}{\left(2 L+L_{2}\right)^{4}} \\
0 \\
0
\end{array}\right) .
$$

Consequently, the $x$ displacement after one stroke is approximated by

$$
\begin{aligned}
x(T)-x(0)= & C\left(\frac{\eta-\xi}{\xi}\right)\left[\frac{L^{3} L_{2}\left(3 L+2 L_{2}\right)}{\left(2 L+L_{2}\right)^{4}}\right] \\
& +o\left(a_{i}^{3}\right)_{i=1-4} .
\end{aligned}
$$

Setting the total length of the swimmer by a constant equal to $c$, i.e., $2 L+L_{2}=c$, we find that (13) has a unique maximum at

$$
L^{*}=c\left(1-\sqrt{\frac{2}{5}}\right), \quad L_{2}^{*}=c\left(2 \sqrt{\frac{2}{5}}-1\right),
$$

which gives an optimal ratio of

$$
\left(\frac{L_{2}}{L}\right)^{*}=\frac{\sqrt{10}-1}{3} \sim 0.721 \text {. }
$$

Remark. In [13] an optimal ratio of 0.747 is given for an efficiency-type criterion. The small gap may be due to the difference in models or the change of the objective function.

\section{NUMERICAL SIMULATIONS}

We solve now the optimal control problem (OCP) numerically, in order to determine the optimal swimming strategy and link ratio. Simulations are performed with the toolbox BOCOP ([19]) that implements a direct transcription method. This approach uses a time discretization to transform the continuous (OCP) into a finite-dimensional optimization problem (nonlinear programming). We refer interested readers to [20] for more details on these methods. We use here an implicit midpoint discretization with 100 to 2500 time steps. Note that this method does not use the PMP. 
TABLE I. Small amplitude $(a=\pi / 20)$.

\begin{tabular}{lccl}
\hline \hline$b$ & $x(T)$ & $L_{2} / L$ & Stroke \\
\hline 0.5 & $2.68 \mathrm{E}-3$ & 0.719 & Diamond \\
$\pi / 5$ & $4.23 \mathrm{E}-3$ & 0.719 & Diamond \\
0.75 & $5.70 \mathrm{E}-3$ & 0.719 & Octagon \\
1 & $7.73 \mathrm{E}-3$ & 0.719 & Octagon \\
$2 \pi / 5$ & $8.42 \mathrm{E}-3$ & 0.717 & Square \\
1.5 & $1.14 \mathrm{E}-2$ & 0.719 & Octagon $(\times 2)$ \\
2 & $1.55 \mathrm{E}-2$ & 0.719 & Octagon $(\times 2)$ \\
\hline \hline
\end{tabular}

As stated in (OCP), the criterion is to maximize the total displacement along the $x$ axis over a fixed time $T$. The initial state of the swimmer is set as $x(0)=y(0)=\theta_{2}(0)=0$, with the final conditions $y(T)=\theta_{2}(T)=0$. The initial shape angles are left free, with the periodicity conditions $\beta_{i}(0)=\beta_{i}(T), i=$ 1,3 . We set the total length $c=4$ for an easier comparison with the classical Purcell swimmer ( $\left.L=1, L_{2}=2\right)$.

We explore different values for the bounds $a, b$ on the shape angles and deformation speed and see their influence on the optimal stroke and link ratio. For practical applications, the values for $a$ and $b$ should reflect the physical characteristics of the studied swimmer. It should be pointed out that the period of the optimal stroke is not known a priori. We arbitrarily set $T=1$ in the first set of simulations, and $T=25$ when studying the larger amplitudes. In the latter case we find that the swimming strategy consists of a periodic sequence of identical strokes, as previously observed in [8].

\section{A. Small amplitudes, influence of speed limits}

We start with small amplitudes by setting $a=\pi / 20$ and solve (OCP) for different values of the speed limit $b$. Here we set $T=1$ and use 250 time steps for the discretization. Optimizations take about $1 \mathrm{~min}$ on a standard laptop. Results are given in Table I, with the phase portraits for the shape angles $\beta_{1}, \beta_{3}$ on Fig. 3.

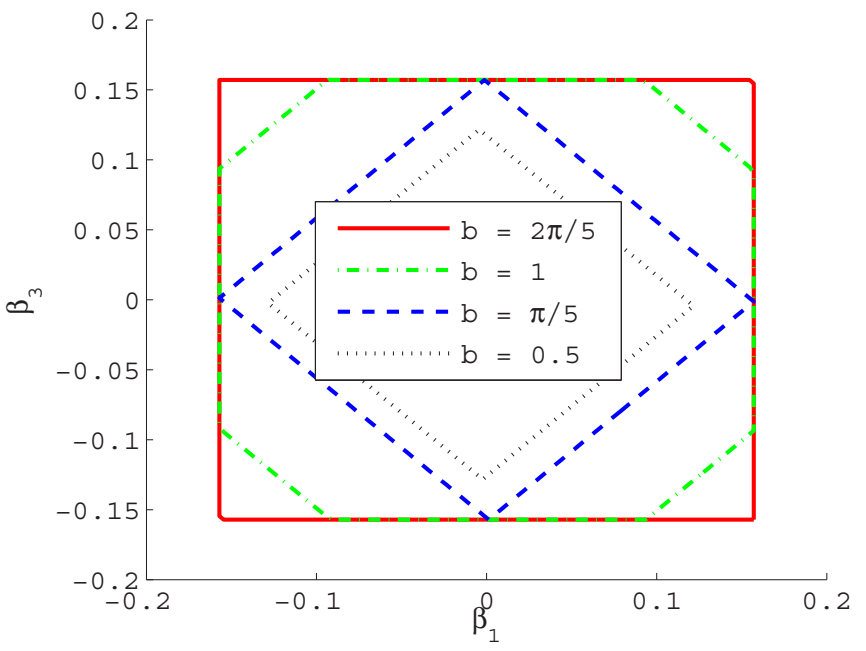

FIG. 3. (Color online) Phase portraits of the strokes for small amplitudes, $a=\pi / 20$. The shapes observed are consistent with the discussion in Sec. II.
TABLE II. Optimal swimmer vs Purcell swimmer.

\begin{tabular}{lcclcc}
\hline \hline $\mathrm{b}$ & $x(T)$ & $L_{2} / L$ & Stroke & $x_{\text {Purcell }}(T)$ & Gain $(\%)$ \\
\hline$p i / 3$ & $1.17 \mathrm{E}-2$ & 0.717 & Diamond & $7.373 \mathrm{E}-3$ & 51 \\
$2 \pi / 3$ & $4.57 \mathrm{E}-2$ & 0.708 & Diamond & $2.848 \mathrm{E}-2$ & 60 \\
$\pi$ & $7.82 \mathrm{E}-2$ & 0.699 & Octagon & $4.806 \mathrm{E}-2$ & 63 \\
$4 \pi / 3$ & $8.80 \mathrm{E}-2$ & 0.695 & Square & $5.359 \mathrm{E}-2$ & 64 \\
\hline \hline
\end{tabular}

First we observe that the optimal ratio $L_{2} / L$ is very close to its theoretical value of 0.721 from (15), regardless of $b$. The speed bound does, however, have an influence on the shape of the optimal stroke and its displacement. Displacement increases with higher speeds, and we find the following empirical relation between $b$ and the stroke shape, confirmed by simulations with other values of $a$ :

(i) for $b<4 a / T$, diamond stroke, which touches the bound $a$ for the limit case $b=4 a / T$;

(ii) for $4 a / T<b<8 a / T$, octagonal stroke;

(iii) for $b=8 a / T$, classical Purcell stroke (square);

(iv) for $b>8 a / T$, sequence of several strokes.

The three strokes observed (diamond, octagon, square) match the discussion from Sec. II. They include only diagonal lines (bang arcs saturating the speed limit $b$ ) and horizontal and vertical lines (constrained arcs for the amplitude limit $a$ ). Note also that the square and diamond strokes are particular cases of the octagonal one, by setting the appropriate arc lengths to 0 .

Remark. This empirical relation can also be interpreted in terms of the period $T$, with the two limit values $T=8 a / b$ for the Purcell stroke and $T=4 a / b$ for the diamond touching $a$.

\section{B. Comparison with the classical Purcell swimmer}

Now we compare the performance of the optimal swimmer with respect to the classical Purcell swimmer defined by $L=1, L_{2}=2$, meaning a ratio of 2 . For this comparison we set $a=\pi / 6$ (thus, a stroke amplitude of $\pi / 3$ ) and $b=$ $\pi / 3,2 \pi / 3, \pi, 4 \pi / 3$ and $T=1$. The optimization for the Purcell swimmer is done by setting $L=1$ instead of letting it be free. The results are summed up in Table II and Fig. 4. We

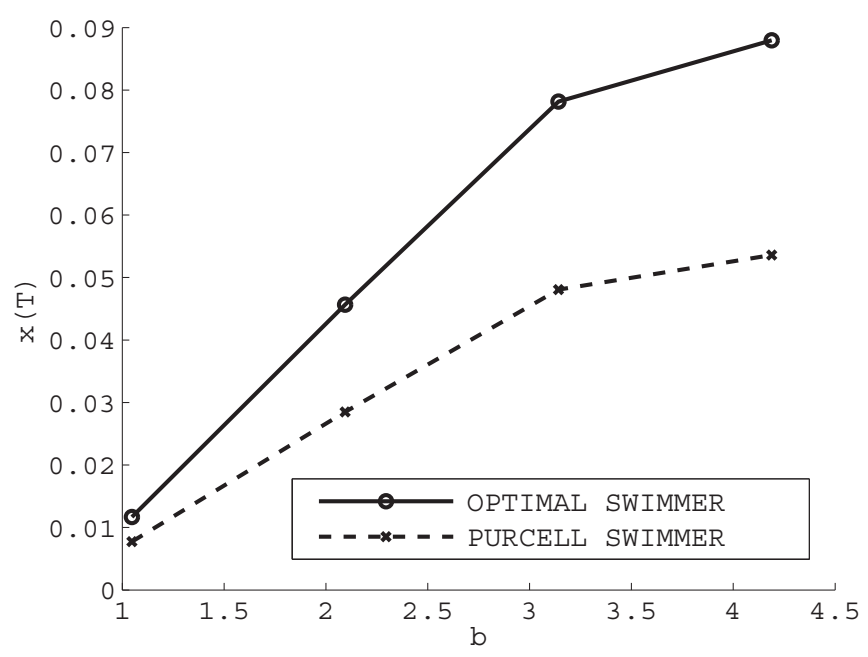

FIG. 4. Displacement for the optimal/Purcell swimmer. 
TABLE III. Larger amplitudes: Optimal link ratio and stroke. Solutions become unconstrained about $a=1.95$.

\begin{tabular}{llll}
\hline \hline$a$ & $x(T)$ & $L_{2} / L$ & \multicolumn{1}{c}{ Stroke } \\
\hline$\pi / 20$ & 0.192 & 0.719 & Octagon $\times 26$ \\
$\pi / 10$ & 0.384 & 0.712 & Octagon $\times 13$ \\
$\pi / 6$ & 0.593 & 0.697 & Octagon $\times 7$ \\
0.75 & 0.811 & 0.676 & Octagon $\times 5$ \\
$\pi / 3$ & 1.088 & 0.660 & Octagon $\times 4$ \\
1.25 & 1.266 & 0.660 & Octagon $\times 4$ \\
1.5 & 1.263 & 0.660 & Octagon $\times 3$ \\
1.75 & 1.329 & 0.667 & Octagon $\times 3$ \\
$2 \pi / 3$ & 1.335 & 0.667 & Unconstrained $\times 3$ \\
2.5 & 1.335 & 0.667 & Unconstrained $\times 3$ \\
\hline \hline
\end{tabular}

see that the shape of the stroke matches the empirical law and that the optimal link ratio stays close to its theoretical value. We also observe a consistent gain in displacement that seems to increase with the speed limit, up to $64 \%$ for the classical Purcell stroke (square).

\section{Large amplitudes, influence of angle limits}

Now we study the influence of the maximal amplitude of the stroke, set by the bound $a$. In this last part we set the deformation speed limit $b=1$ to focus on the amplitude. Since we would like to study only the true optimal strokes, whose period is not known, we also take a longer final time $T=25$. We expect to obtain trajectories that exhibit a sequence of several identical strokes with a period $T^{*}<T$. The number of time steps is raised accordingly to 2500 , which increases the computational time up to half an hour. Another way of finding the optimal stroke directly could be to leave the final time $T$ free in the optimization, while maximizing the average speed of the stroke $x(T) / T$ instead of the displacement $x(T)$.

The results are illustrated in Table III and Figs. 5 and 6. First, the simulations confirm that the optimal strategy is a periodic sequence of identical strokes. The shape of the optimal

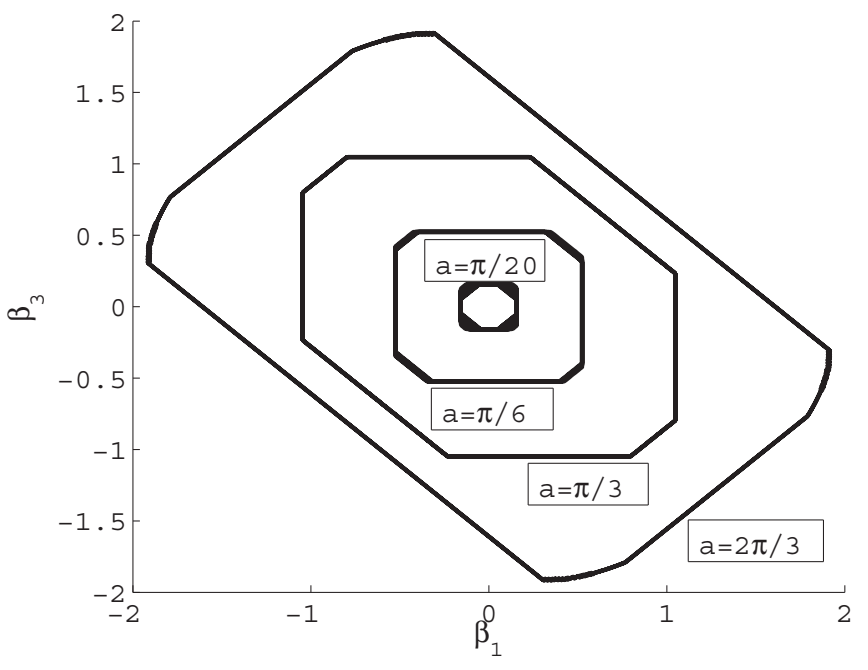

FIG. 5. Larger amplitudes, phase portrait (each trajectory consists in several superposed strokes).

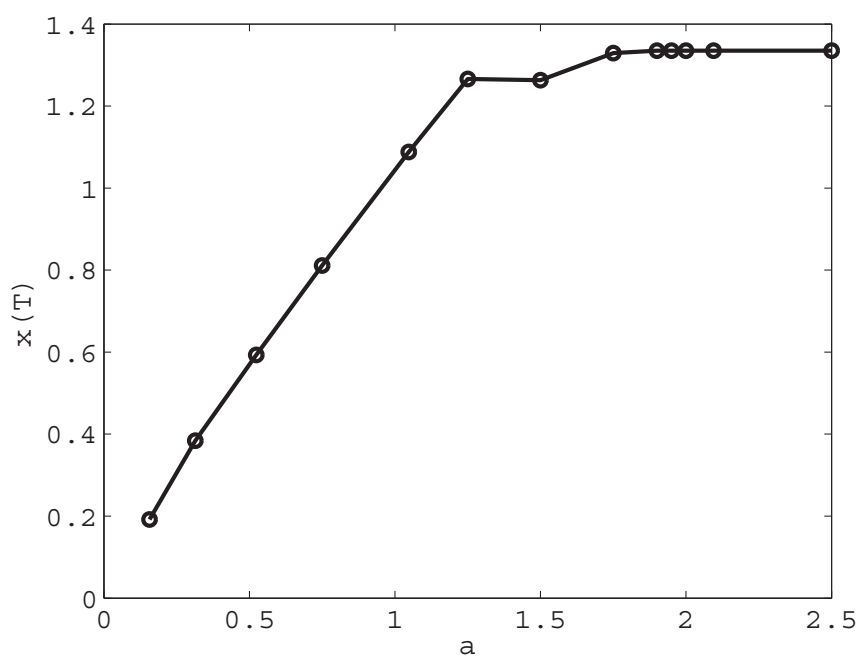

FIG. 6. Larger amplitudes, overall displacement. For $a=1.5$ the optimization converged to a local solution, since displacement is expected to be an increasing function of $a$.

stroke is always octagonal until it becomes unconstrained for very large values of $a$. We observe that the central symmetry observed for small amplitudes is lost for larger $a$; however, symmetry with respect to both diagonal axes still holds as expected.

In the unconstrained case, we see arcs that are neither bang arcs (diagonal) or constrained arcs (horizontal and vertical), but rather appear as smooth curves (see Fig. 5). These are characteristic of so-called singular arcs, namely the case where $\left\langle p, g_{i}(z)\right\rangle=0$ in the PMP. More details on the analysis of singular arcs can be found in [17]; unfortunately, here the complexity of the $g_{i}$ makes further study quite difficult.

The total displacement $x(T)$ increases with $a$, first almost linearly when $a<\pi / 3$ (see Fig. 6). From $a \approx 1.95$ and above, we obtain the same, unconstrained solution. The improvement in displacement appears to be marginal between $a=\pi / 3$ and the unconstrained case. Note that since the displacement is expected to be a monotone increasing function of $a$, we see that for $a=1.5$, the optimization converged to a local solution.

The optimal ratio $L_{2} / L$ shows a steady decrease with $a$, starting quite close to the value 0.721 computed for small amplitudes, then seemingly reaching a limit value of $2 / 3$ in the unconstrained case (i.e., $L=1.5, L_{2}=1$ ). We recall that the classical Purcell swimmer has a link ratio of $2\left(L=1, L_{2}=2\right)$.

\section{CONCLUSION}

This study is devoted to the optimization of the link ratio of the three-link swimmer for maximal displacement. We provide an estimate of the displacement based on an expansion for small deformations, which gives a theoretical optimal link ratio. Numerical simulations are consistent with this theoretical ratio for small amplitudes of deformation. We also observe that the optimal ratio changes for large amplitudes of deformation, with a limit value of 0.667 in the unconstrained case versus a theoretical ratio of 0.721 obtained for small amplitudes of deformation. For an amplitude of $\pi / 3$, the 
displacement gain is about $60 \%$ compared with the classical Purcell swimmer design. A possible continuation of this work is the comparison of different objective functions, such as speed or efficiency.
[1] J. Lighthill, Mathematical Biofluiddynamic (Society for Industrial and Applied Mathematics, Philadelphia, 1975).

[2] C. Brennen and H. Winet, Fluid mechanics of propulsion by cilia and flagella, Annu. Rev. Fluid Mech. 9, 339 (1977).

[3] E. Lauga and T. R. Powers, The hydrodynamics of swimming microorganisms, Rep. Prog. Phys. 72, 096601 (2009).

[4] R. Dreyfus, J. Baudry, M. L. Roper, M. Fermigier, H. A. Stone, and J. Bibette, Microscopic artificial swimmers, Nature (London) 437, 862 (2005).

[5] J. Gray and J. Hancock, The propulsion of sea-urchin spermatozoa, J. Exp. Biol. 32, 802 (1955).

[6] F. Alouges, A. DeSimone, L. Giraldi, and M. Zoppello, Self-propulsion of slender micro-swimmers by curvature control: N-link swimmers, Int. J. Nonlinear Mech. 56, 132 (2013).

[7] R. S. Berman, O. Kenneth, J. Sznitman, and A. M. Leshansky, Undulatory locomotion of finite filaments: Lessons from Caenorhabditis elegans, New J. Phys. 15, 075022 (2013).

[8] L. Giraldi, P. Martinon, and M. Zoppello, Controllability and optimal strokes for N-link micro swimmer, in Proceedings of the 52nd Annual IEEE Conference on Decision and Control (CDC), 2013 (IEEE, Piscataway, NJ, 2013), pp. 3870-3875.

[9] E. M. Purcell, Life at low Reynolds number, Am. J. Phys. 45, 3 (1977).

[10] L. E. Becker, S. A. Koehler, and H. A. Stone, On self-propulsion of micro-machines at low Reynolds number: Purcell's three-link swimmer, J. Fluid Mech. 490, 15 (2003).
[11] J. E. Avron and O. Raz, A geometric theory of swimming: Purcell's swimmer and its symmetrized cousin, New J. Phys. 10, 063016 (2008).

[12] R. Montgomery, A Tour of Subriemannian Geometries, Their Geodesics and Applications (American Mathematical Society, Providence, RI, 2002).

[13] D. Tam and A. E. Hosoi, Optimal stroke patterns for Purcell's three-link swimmer, Phys. Rev. Lett. 98, 068105 (2007).

[14] A. Najafi and R. Golestanian, Simple swimmer at low Reynolds number: Three linked spheres, Phys. Rev. E 69, 062901 (2004).

[15] V. G. Boltyanskii, R. V. Gamkrelidze, and L. S. Pontryagin, Towards a theory of optimal processes, Rep. Acad. Sci. USSR 110, 19 (1956).

[16] A. A. Agrachev, Nonlinear and Optimal Control Theory (Springer Verlag, Berlin, 2008).

[17] E. Trelat, Contrôle Optimal: Théorie and Applications, Collection Mathématiques Concrètes (Vuibert, Paris, 2005).

[18] J. M. Coron, Control and Nonlinearity (American Mathematical Society, Providence, RI, 2007).

[19] F. Bonnans, D. Giorgi, S. Maindrault, P. Martinon, and V. Grelard, Bocop-A Collection of Examples, Technical Report, INRIA, http://www.bocop.org, 2014.

[20] J. Betts, Practical Methods for Optimal Control Using Nonlinear Programming, Advances in Design and Control (Society for Industrial and Applied Mathematics, Philadelphia, 2001). 